\title{
Fatal (Computer Sense)
}

National Cancer Institute

\section{Source}

National Cancer Institute. Fatal(Computer Sense). NCI Thesaurus. Code C71755.

Resulting in termination of the program. 